\title{
Actividades de la campaña del Día Mundial de la Higiene de Manos en el Hospital Juárez de México 2019
}

\section{Activities of the World Hand Hygiene Day campaign at Hospital Juárez de México 2019}

\author{
Oscar Sosa-Hernández ${ }^{*}$, Bernardina Matías-Téllez ${ }^{1}$ Luis A. Gorordo-Delsol², Edith González-Vázquez³, \\ Yefté E. López-Silva ${ }^{4}$ y Mónica A. Cureño-Díaz ${ }^{5}$ \\ ${ }^{1}$ Unidad de Vigilancia Epidemiológica Hospitalaria; ${ }^{2}$ Unidad de Cuidados Intensivos de Adultos; ${ }^{3}$ Infectología Pediátrica; ${ }^{4}$ División de Calidad de \\ la Atención; ${ }^{5}$ Dirección de Planeación Estratégica. Hospital Juárez de México, Ciudad de México, México
}

\begin{abstract}
Resumen
Introducción: Cada año, la Organización Mundial de la Salud promueve la campaña "Salva vidas: Límpiese las manos», para crear conciencia sobre la importancia de la higiene de manos para la prevención de infecciones y la resistencia antimicrobiana. Material y métodos: Para este trabajo se diseñó un estudio de tipo transversal, observacional, descriptivo y retrospectivo. Para el porcentaje de apego de lavado e higiene de manos se utilizaron las bases de datos de monitoreo de sombra del periodo que comprende de junio de 2018 a mayo de 2019, de la Unidad de Vigilancia Epidemiológica Hospitalaria. Resultados: El apego al lavado e higiene de manos muestra un incremento gradual, pasando del $46 \%$ en junio de 2018 al 59\% en mayo de 2019. Para la obtención de estas mediciones de apego se han realizado un total de 28,710 observaciones a través de estudios de sombra. En cuanto al apego al lavado e higiene de manos por categoría, en promedio, el personal de enfermería tiene un apego del $64 \%$, con un mínimo del $54 \%$ y un máximo del $72 \%$; otro personal de salud tiene un apego promedio del $51 \%$, con un mínimo del 34\% y un máximo del 62\%; y el personal médico tiene un apego promedio del $40 \%$, con un mínimo del $29 \%$ y un máximo del $46 \%$. Conclusiones: La participación de los diferentes servicios en el plan de capacitación de higiene de manos es conveniente y necesaria, pues las áreas deben crear lazos de trabajo a fin de generar y fortalecer estrategias y acciones que tengan impacto en todo el hospital.
\end{abstract}

Palabras clave: Higiene de las manos. Prevención de enfermedades. Control de enfermedades transmisibles.

\section{Abstract}

Introduction: Every year the World Health Organization promotes the campaign "Save lives: Clean hands", to raise awareness about the importance of hand hygiene for the prevention of infections and antimicrobial resistance. Material and methods: For this work, a transversal, observational, descriptive and retrospective study was designed. For the percentage of hand washing and hygiene attachment, the shadow monitoring databases for the period from June 2018 to May 2019 of the Hospital Epidemiological Surveillance Unit were used. Results: The attachment to washing and hand hygiene shows a gradual increase going from 46\% in June 2018, to 59\% in May 2019. To obtain these measurements of attachment, a total of 28,710 observations have been made through studies of shadow. The adherence to washing and hand hygiene by category, on average the

Correspondencia:

*Oscar Sosa-Hernández

E-mail: dr.oscar.sh@gmail.com
Disponible en internet: 10-03-2020 Rev Hosp Jua Mex. 2020;87(1):17-20

www.revistahospitaljuarez.com 1405-9622/@ 2019 Sociedad Médico-Quirúrgica del Hospital Juárez de México, A.C. Publicado por Permanyer. Este es un artículo open access bajo la licencia CC BY-NC-ND (http://creativecommons.org/licenses/by-nc-nd/4.0/) 
nursing staff has an attachment of $64 \%$, with a minimum of $54 \%$ and a maximum of $72 \%$; other health personnel have an average attachment of $51 \%$, with a minimum of $34 \%$ and a maximum of $62 \%$; and the medical staff has an average attachment of $40 \%$ with a minimum of $29 \%$ and a maximum of $46 \%$. Conclusions: The participation of the different services in the hand hygiene training plan is convenient and necessary, since the areas must create work ties in order to generate and strengthen strategies and actions that have an impact throughout the hospital.

Key words: Hand hygiene. Disease prevention. Communicable disease control.

\section{Introducción}

Cada año, la Organización Mundial de la Salud (OMS) promueve la campaña «Salva vidas: Límpiese las manos", con el objetivo de crear conciencia en el personal de salud sobre la importancia de la higiene de manos para la prevención de infecciones y la resistencia antimicrobiana ${ }^{1}$. En el año 2018, el lema fue «Está en sus manos, prevenir la sepsis en la atención de la salud", y se realizaron actividades en conjunto con la Unidad de Cuidados Intensivos de Adultos y la Fundación Sepsis México para dar difusión a esta medida hacia diferentes foros ${ }^{2}$.

\section{Día Mundial de la Higiene de Manos 2019}

Para el año 2019, la campaña del Día Mundial de la Higiene de Manos estaba diseñada para incluir a todo el personal a realizar esta medida con el lema "Una atención limpia para todos - está en sus manos» y con las siguientes acciones:

- Profesionales de la salud: Defiendan una atención limpia - está en sus manos.

- Líderes de prevención y control de infecciones: Supervisen los estándares de prevención y control de infecciones - tomen medidas y mejoren prácticas.

- Líderes del centro de salud: ¿Sus instalaciones están a la altura de los estándares de la OMS de higiene de manos y control de infecciones? ¡Participen en la encuesta de la OMS 2019 y tomen medidas!

- Ministerios de Salud: ¿Su país cumple con los estándares de control y prevención de infecciones? Supervisen y actúen para lograr una cobertura de salud universal de calidad.

- Grupos de defensa del paciente: Soliciten una atención limpia - es su derecho.

Cada año, la Unidad de Vigilancia Epidemiológica Hospitalaria (UVEH) lleva a cabo durante una semana actividades que ayuden a fomentar en el personal de salud una cultura de seguridad ${ }^{2}$. Para el año 2019, en la campaña «Una atención limpia para todos - está en sus manos", adaptamos la campaña con nuestro lema institucional «Higiene de manos de todos para todos en el Hospital Juárez de México», por lo que formamos grupos con personal de salud, médico y de enfermería que tiene intervención clínica, directivos y áreas de enlace con asociaciones de apoyo a los pacientes, con la finalidad de promover la importancia de la higiene de manos a otro personal de salud 3 .

El objetivo es describir las actividades de la Campaña del Día Mundial de la Higiene de Manos en el Hospital Juárez de México 2019.

\section{Material y métodos}

Para este trabajo se diseñó un estudio de tipo transversal, observacional, descriptivo y retrospectivo. Se describieron, de manera general, las actividades realizadas durante la Campaña del Día Mundial de la Higiene de Manos, además se describió el porcentaje de apego de lavado e higiene de manos, por lo que se utilizaron las bases de datos de monitoreo de sombra del periodo que comprende de enero a junio de 2019, de la UVEH. Para la descripción de los datos se utilizaron frecuencias simples y porcentajes.

\section{Resultados}

Durante la campaña de 2019, se incluyeron diferentes servicios hospitalarios para realizar la capacitación y concientización dirigidas a personal de salud de nuestra institución. Para esta capacitación se realizaron dos actividades:

- Módulo de capacitación de higiene de manos: consiste en un espacio colocado durante una semana en uno de las áreas de mayor tránsito de nuestra institución, donde se tiene contacto con el personal de diferentes servicios hospitalarios, clínicos, paraclínicos y administrativos.

- Brigadas de capacitación de higiene de manos: son grupos conformados por personal de salud de diferentes áreas, que acudieron a los servicios para capacitar al personal de áreas clínicas y paraclínicas que no podían acudir al módulo por cubrir sus actividades diarias. Las áreas involucradas fueron 


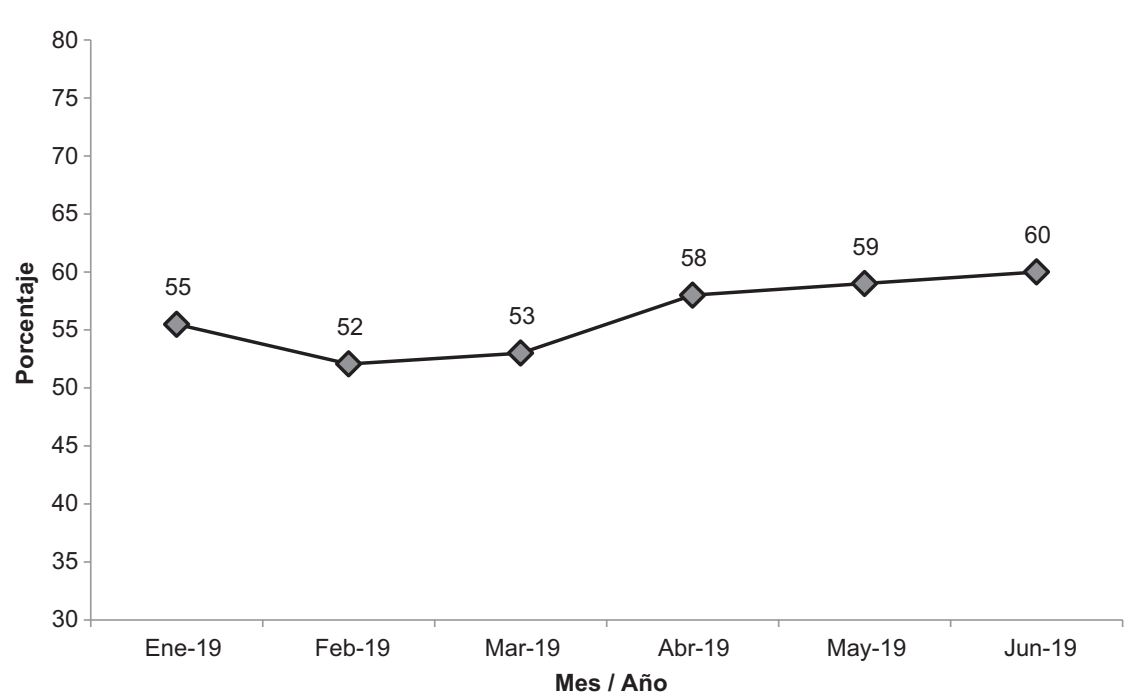

Figura 1. Porcentaje de apego al lavado e higiene de manos de enero a mayo de 2019.

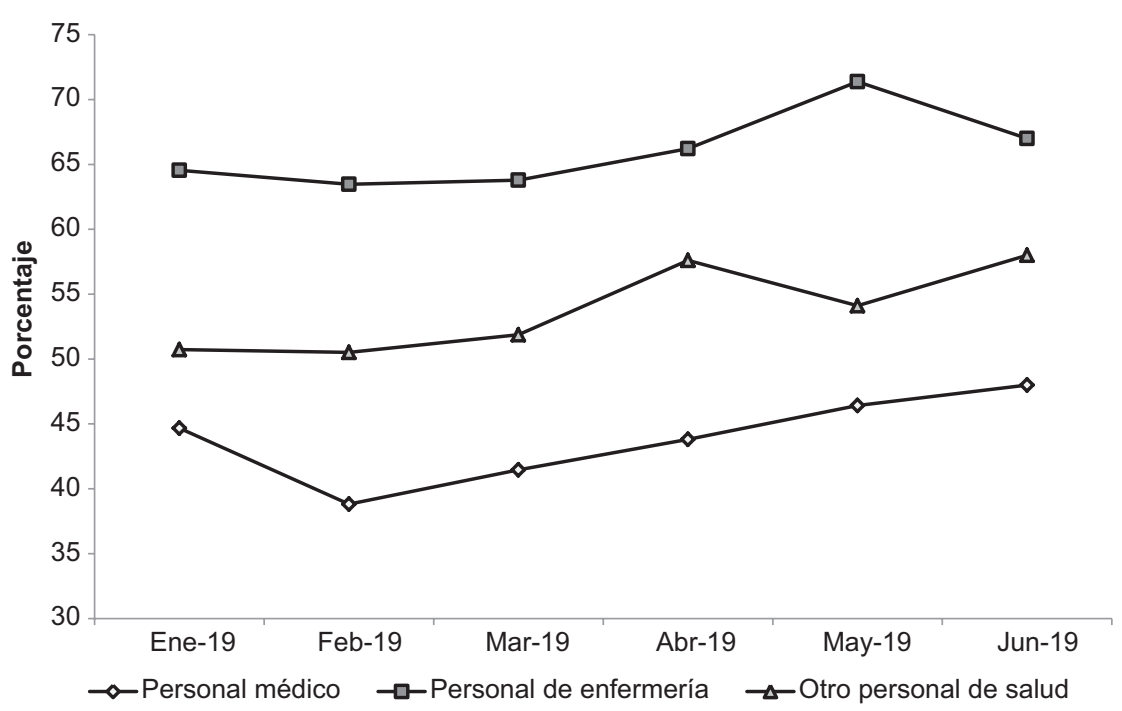

Figura 2. Porcentaje de apego al lavado e higiene de manos por personal de salud de enero a mayo de 2019.

Dirección de Planeación, Unidad de Cuidados Intensivos. De esta forma, el personal de áreas hospitalarias diferentes al de la UVEH tuvo la oportunidad de capacitar y poder experimentar este acercamiento, el cual, en mucha de las ocasiones suele ser desestimado por la sencillez de la higiene de manos.

En total se capacitaron 1,173 trabajadores de la salud, de los cuales, el $46.3 \%$ correspondió a otro personal de salud, el $30.9 \%$ correspondió al personal de enfermería y el $22.8 \%$ correspondió al personal médico.

El apego al lavado e higiene de manos muestra un incremento gradual, pasando del $46 \%$ en junio de 2018 al 60\% en junio de 2019 (Fig. 1). Para la obtención de estas mediciones de apego se han realizado un total de 28,710 observaciones a través de estudios de sombra. Al revisar el apego al lavado e higiene de manos por categoría, en promedio, el personal de enfermería 
tiene un apego del $64 \%$, con un mínimo del $54 \%$ y un máximo del $72 \%$; otro personal de salud tiene un apego promedio del $51 \%$, con un mínimo del $34 \%$ y un máximo del $62 \%$; y el personal médico tiene un apego promedio del $40 \%$ con un mínimo del $29 \%$ y un máximo del $46 \%$ (Fig. 2). Además, se puede observar que los apegos del personal de enfermería y personal médico, en el mes de mayo, son los más altos, esto se adjudica a la capacitación que se realizó en todos los servicios que se realizaron como parte de las actividades del Día Mundial de la Higiene de Manos del 2019.

\section{Discusión}

A partir de estudios experimentales, en 1847, el médico húngaro Ignaz Philipp Semmelweis (18181865), al percatarse de los elevados índices de fiebre puerperal, postuló la interacción entre lavado de las manos y la fiebre puerperal. De modo que instruyó la obligatoriedad de la higiene de las manos con solución clorada entre la atención de cada paciente. En este momento, se evidenció la reducción drástica de la tasa de mortalidad materna 4 .

Al comparar el apego al lavado e higiene de manos de nuestra institución con los reportes en artículos, encontramos que los porcentajes bajos a esta medida son reportados en diferentes países. Lambe, et al. realizaron una revisión sistemática del apego en unidades de cuidados intensivos. Describieron que, en países con ingresos económicos elevados, el apego fue del $64.5 \% \mathrm{y}$, en países con ingresos bajos, puede llegar hasta el 9.1\%. En terapias intensivas neonatales, el apego mostrado fue del $67 \%$, en terapias intensivas pediátricas, del $41.2 \%$ y en UCIA, del $53.8 \%$. Por categoría, describen que el apego de otro tipo de personal fue del $53.8 \%$, del personal de enfermería, del $43.4 \%$ y del personal médico, del $32.6 \% 5$.

Muller, et al. describieron el apego al lavado e higiene de manos en una unidad de urgencias en un hospital en Toronto, Canadá, donde el apego fue del 29\%: el personal de enfermería mostró un apego del $68 \%$, el personal médico, del $14 \%$ y otro personal, del $14 \%$. Al describir el apego de acuerdo a los cinco momentos de higiene de manos, el momento con un mayor apego fue «después de tocar al paciente», con un 37\%, y el de menor apego fue «antes de realizar una tarea limpia 0 aséptica", con un $10 \%{ }^{6}$.

\section{Conclusiones}

La participación de los diferentes servicios con la UVEH en el plan de capacitación de la higiene de manos es conveniente y necesaria, pues las áreas deben crear lazos de trabajo a fin de generar y fortalecer estrategias y acciones que tengan impacto en todo el hospital. Sin duda, es necesario que las instituciones de salud cuenten con profesionales de la salud empeñados en la adopción de estrategias de control de las infecciones y, en este sentido, el programa de higiene de manos representa el recurso más sencillo y efectivo de adoptar.

\section{Conflicto de intereses}

Los autores declaran no tener conflicto de intereses alguno.

\section{Financiamiento}

Los autores no recibieron patrocinio para llevar a cabo este artículo.

\section{Responsabilidades éticas}

Protección de personas y animales. Los autores declaran que los procedimientos seguidos se conformaron a las normas éticas del comité de experimentación humana responsable y de acuerdo con la Asociación Médica Mundial y la Declaración de Helsinki.

Confidencialidad de los datos. Los autores declaran que en este artículo no aparecen datos de pacientes.

Derecho a la privacidad y consentimiento informado. Los autores declaran que en este artículo no aparecen datos de pacientes.

\section{Bibliografía}

1. Peters A, Kilpatrick C, Pittet D. "Clean care for all - It's in your hands": The May $5^{\text {th }}, 2019$ World Health Organization SAVE LIVES: Clean Your Hands campaign. International Journal of Infectious Diseases. 2019;82:135-6.

2. Sosa-Hernández $O$, Gorordo-Delsol LA. Importancia de la higiene de manos para prevenir la sepsis en la atención de la salud. Med Crit. 2018;32(5):295-6.

3. Sosa-Hernández $\mathrm{O}$. De todos para todos, la higiene de manos en el Hospital Juárez de México, campaña del Día Mundial de Higiene de Manos 2019. Rev Hosp Jua Mex. 2019;86(2):68-9.

4. Miranda M, Navarrete L. Semmelweis y su aporte científico a la medicina: un lavado de manos salva vidas. Rev Chil Infect. 2008;25(1):54-7.

5. Lambe KA, Lydon S, Madden C, Vellinga A, Hehir A, Walsh M, et al. Hand Hygiene Compliance in the ICU: A Systematic Review. Crit Care Med. 2019 Jun 18.

6. Muller MP, Carter E, Siddiqui N, Larson E. Hand Hygiene Compliance in an Emergency Department: The Effect of Crowding. Acad Emerg Med. 2015 Oct;22(10):1218-21. 